\title{
Agua en territorios comunales: gestión del riego en el valle del río Javita, provincia de Santa Elena
}

\section{Water in communal territories: irrigation management in the Javita river valley, Santa Elena province}

\author{
Pablo Raúl Velasco Andrade ${ }^{*}$, Christian Tamayo Ortiz ${ }^{2}$ \\ ${ }^{1}$ Comuna Olón, Santa Elena. \\ ${ }^{2}$ Universidad Central del Ecuador, Facultad de Ciencias Agrícolas, Carrera Ingeniería Agronómica Jerónimo Leiton y Av. La Gasca \\ s/n, Ciudadela Universitaria. Quito. 170521. Ecuador
}

凶 Autor para correspondencia: pablorvelasco@gmail.com

\begin{abstract}
Resumen
Este trabajo se propuso analizar la gestión del riego en el valle del río Javita, provincia de Santa Elena, en el contexto de la puesta en marcha del trasvase Chongón San Vicente. Se trabajó con métodos participativos, recabando información de una muestra de productores. Los efectos del trasvase Chongón San Vicente se revelaron insuficientes en cuanto a eficacia, pertinencia, entorno institucional, aspectos sociales y culturales, y ambientales. Como casos de estudio, se hizo el análisis de cinco tipos de sistemas de producción. En todos los casos, los ingresos de actividades extra finca son superiores a lo obtenidos de actividades agropecuarias. Los sistemas de producción agropecuaria que mejores ingresos obtienen se dedican exclusivamente a cultivos bajo riego. La investigación determina como marco orientador que: la organización del riego se haga por grupos familiares; la administración, operación y mantenimiento corra a cargo de las juntas de riego; el reparto de agua se efectúe según la necesidad de los cultivos; el método de aplicación sea por goteo; la instalación vaya en función del ahorro de agua; los cultivos sean de preferencia de ciclo corto y transitorio; y, el destino de la producción se oriente al mercado regional o nacional. Para que el riego se convierta en un elemento dinamizador de la economía local, no basta con obras de infraestructura. Es indispensable que el Estado invierta en un programa de desarrollo rural inclusivo, equitativo y eficiente.
\end{abstract}

Palabras clave: recursos hídricos; campesinado; manejo de recursos hídricos; comunas y recursos hídricos.

\begin{abstract}
This work was proposed to analyze the management of irrigation in the Javita River Valley, Province of Santa Elena, in the context of the implementation of the San Vicente Chongón Transfer. The work was done with participatory methods, gathering information from a sample of farmers in the area. The effects of the Chongón San Vicente water transfer were insufficient in terms of efficiency, relevance, institutional environment, social, cultural, and environmental aspects, according to the opinion of the participants. As case studies, five types of production systems were analyzed. In all cases, the income from extra-farm activities is higher than that obtained from agricultural activities. The agricultural production systems that obtain the best income are those dedicated exclusively to crops under irrigation. The research determines as a guiding framework that: the organization of irrigation is done by family groups; administration, operation and maintenance are allowed by the irrigation boards; the distribution of water is carried out according to the need of the crops; the method of application is drip; the installation depends on the water saving; the crops are preferably short and transient cycle; and, the destination of the production is oriented to the regional or national market. For irrigation to become a dynamic element of the local economy, infrastructure works are not enough. It is essential that the government invest in an inclusive, equitable and efficient rural development program.
\end{abstract}

Keywords: water resources; peasantry; water resources management; infrastructure and irrigation; communes and water resources 


\section{Introducción}

La necesidad creciente de dotar de agua al territorio de la península de Santa Elena, ubicada en la zona sur de la costa ecuatoriana, obligó a las entidades oficiales a encontrar una fuente segura, suficiente y de calidad como el río Daule, así como a construir estructuras con capacidad de captación, embalse, regulación, conducción y distribución. De allí surgieron el Proyecto Hidráulico Acueducto de Santa Elena y el Trasvase Chongón San Vicente, una de cuyas áreas a ser beneficiadas con riego es el valle del río Javita (GADPSE, 2016, p. 24). No obstante, hasta la presente fecha, el valle del río Javita, zona central norte de la provincia de Santa Elena, muestra severas deficiencias de agua para riego y consumo humano, debido a que su territorio tiene el déficit hídrico más elevado del país y al hecho que desde hace varias décadas, las entidades competentes, no han podido culminar satisfactoriamente el trasvase de aguas desde la vecina cuenca del Guayas hasta el territorio peninsular, a pesar de las considerables inversiones efectuadas.

En efecto, hasta la fecha, no se han efectuado los estudios y diseños de riego y drenaje parcelario para el valle del río Javita, aunque ya se ha construido un sistema de conducción y los reservorios en los diversos territorios comunales y se han instalado pequeños sistemas de riego basados en ciertos elementos de la infraestructura ya construida. En estas condiciones, la población se ha visto obligada a trabajar en diversas actividades productivas y no únicamente en la agricultura. Varios elementos han alentado la existencia de la pluriactividad en la zona estudiada: el exiguo ingreso agrícola; el deficiente acceso a la tierra y al servicio de riego; la inestabilidad de la agricultura que depende de la estacionalidad de las precipitaciones; la existencia de oportunidades de empleo en otros sectores y regiones, entre otros factores (Bermeo, 2017, p. 51). La expectativa que creó el trasvase Chongón San Vicente fue la de mejorar las condiciones de vida de más de 80.000 personas, contribuyendo al desarrollo en la producción agropecuaria de varias comunidades rurales, cubriendo de manera progresiva 7.700 hectáreas, permitiendo generar empleos e incrementar la producción (Empresa Pública del Agua, 2014, p. 51).

En la zona en estudio, el régimen comunal vigente se identifica con un tipo de asentamiento nucleado, dentro del cual las familias han venido manteniendo relaciones sociales fundamentalmente de parentesco y en el que están integrados todos sus miembros a través de vínculos familiares y canales institucionalizados de solidaridad, cooperación y ayuda mutua (Álvarez, 2002, p. 11). Las comunas, juntas de agua para consumo humano, asociaciones de productores y juntas de regantes constituyen el núcleo de los procesos de organización social en la zona. Además, se evidencia que la experiencia organizativa en actividades de riego y drenaje ha sido prácticamente inexistente, que el reparto del escaso volumen de agua ha sido marcado por la ineficiencia y la inequidad y que la expectativa social por los beneficios del trasvase es elevada. En esa línea de análisis, el sistema de riego, más allá de constituir una obra de la ingeniería, viene a ser una construcción colectiva de grupos humanos que buscan establecer las maneras de acceder al agua, con los derechos correspondientes, así como el establecimiento de reglas y obligaciones que todos deben acatar (Apollin \& Eberhart, 1998, p. 9).

Este artículo resume los resultados de una investigación de campo respecto de los siguientes elementos: la percepción de los productores locales en relación con los efectos que hasta el momento ha tenido el trasvase Chongón Santa Elena en su territorio; analiza las características de los sistemas de producción campesinos existentes en el territorio en estudio, y también interroga sobre los principales contenidos que debería contener un plan de gestión de riego que favorezca el desarrollo de la agricultura campesina. Esta investigación revela la necesidad de que las entidades encargadas de la implementación del trasvase Chongón San Vicente pongan en marcha un plan de acción que revierta los resultados obtenidos hasta la presente fecha, modificando su política de intervención para lograr la generación de beneficios económicos y sociales directos para la población campesina, tales como el incremento significativo de nuevas áreas de riego que asegure la puesta en marcha de nuevos emprendimientos productivos agropecuarios, en el marco de un programa de desarrollo rural inclusivo, equitativo y eficiente.

\section{Materiales y métodos}

\section{Área de estudio}

Santa Elena es la provincia más joven del país. Situada en el litoral del Ecuador, ocupa un territorio de 
unos $3.700 \mathrm{~km}^{2}$. El déficit hídrico que soporta este territorio se sitúa entre 500 y 1.300 mm/año (GADPSE, 2016, p. 80). La cuenca del río Javita, ubicada en una microrregión seca, se inserta en el territorio de la parroquia Colonche y alberga en su interior a unas quince comunas jurídicas. Es en este territorio en donde se ha instalado el trasvase Chongón San Vicente (Figura 1).

El valle del río Javita posee remanentes de bosque seco y bosque de garúa, correspondiente al bioma Bosque Seco Tumbesino; posee suelos frágiles, procesos erosivos y pérdida de biodiversidad; los procesos de salinización, sedimentación y erosión crecen peligrosamente debido en buena medida a prácticas inadecuadas de manejo del agua de riego; el déficit hídrico trae como efecto la dependencia de cuencas vecinas; los eventos extremos como sequía e inundación causan impactos significativos (GADPSE, 2016, p. 20).

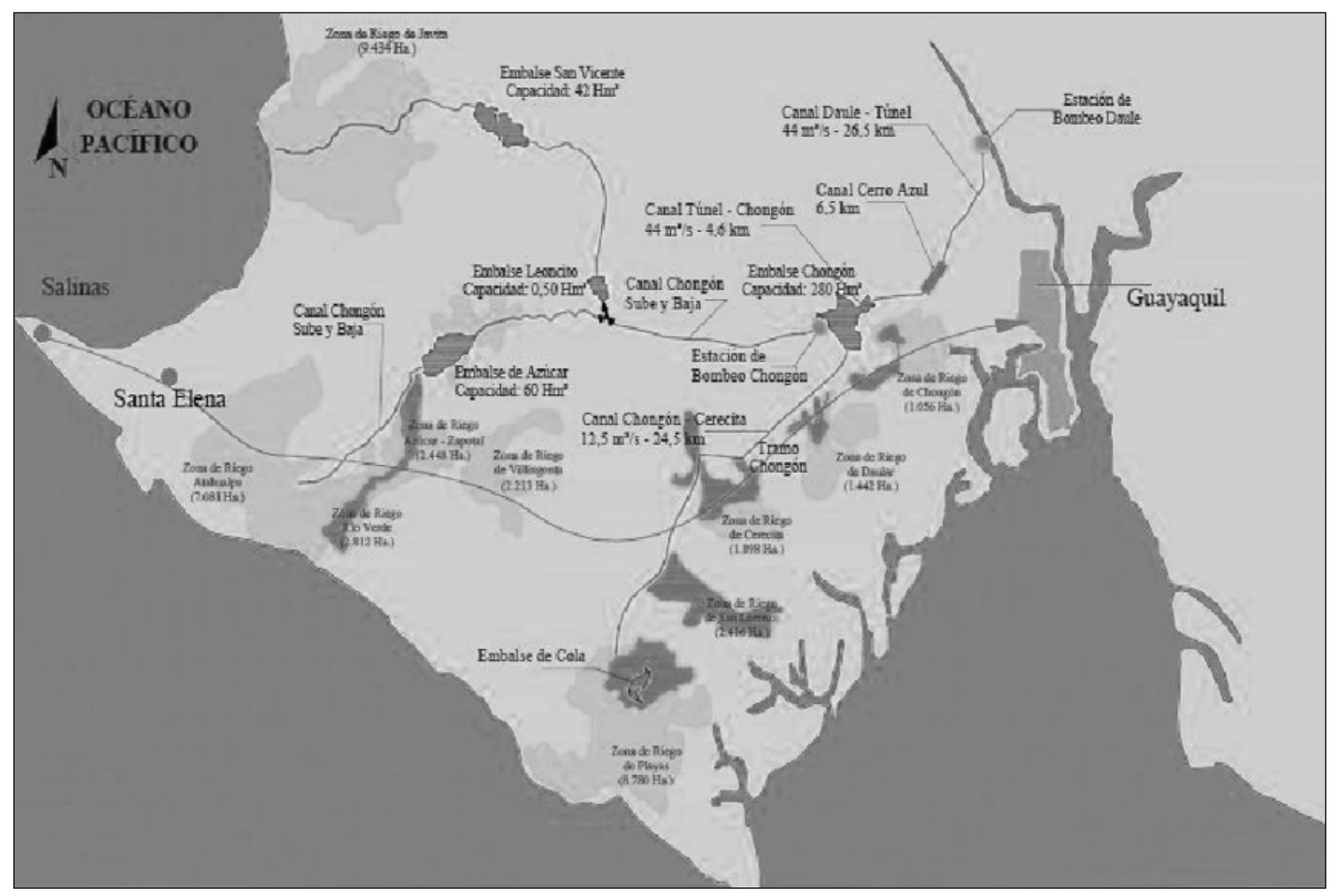

Figura 1. Trasvase de aguas de la cuenca del Daule a Santa Elena.

\section{Metodología}

El estudio de la gestión del riego en el territorio del valle del río Javita fue efectuado considerando al enfoque sistémico como marco conceptual. Para analizar integralmente un sistema de riego desde esta concepción, es necesario abordar el conjunto de sus componentes, así como sus interacciones. Es fundamental percatarse de las consecuencias que un cambio en cualquiera de sus elementos acarrea para el total, es decir para el conjunto del sistema; y es imprescindible utilizarlo para comprender los sistemas de riego (Anten \& Williet, 2000, p. 6).

En primer lugar, se abordó el análisis de los efectos del trasvase Chongón San Vicente en el territorio del valle del río Javita, tomando en consideración el hecho de que esta obra aún se encuentra en proceso de construcción. Consistió en levantar la información sobre la percepción de una muestra de 150 campesinos comuneros respecto de los efectos del trasvase. Se utilizó la técnica de talleres grupales, 
así como la entrevista estructurada. El tamaño muestral se obtuvo calculando la superficie promedio de la que disponen los socios de los once territorios comunales, misma que según el GADPSE (2016, p. 82 ) es de 16,88 ha, en relación con la superficie a ser regada con aguas del trasvase, que es de 4,28 ha. De esta manera, se obtuvo un tamaño de la población a ser muestreada de 245. Se aplicó la fórmula de población finita para calcular la muestra estadística, con un nivel de confianza del 95\% y un margen de error del 5\% determinándose en $\mathrm{n}=150$ el tamaño de la muestra obtenido estadísticamente.

En segundo lugar, se realizó una caracterización de los sistemas de producción campesinos, a través de la aplicación de un formato estructurado a una muestra razonada, no probabilística, de comuneros que viven en el territorio. Se aplicó el instrumento hasta lograr consistencia en la información de cada uno de los casos de estudio, llegándose a un total de 148 productores.

Finalmente, se aplicó un tercer instrumento a la muestra estadística determinada, sobre la base de lo cual se formularon los ejes principales de un plan de gestión del riego que se oriente a favorecer los intereses y demandas de la agricultura campesina en la zona de estudio.

La consulta a los participantes se desarrolló sobre la base de una metodología participativa, basada en la técnica de emoticones -carita feliz, neutra y triste- con campesinos provenientes de once comunas jurídicas de la parroquia Colonche. Al igual que en el primer componente, se utilizó la técnica de talleres grupales, así como la entrevista estructurada.

\section{Resultados y discusión}

\subsection{Efectos del trasvase}

Con base en la revisión bibliográfica se definieron cinco criterios con sus respectivos indicadores para abordar el estudio de los efectos del trasvase: eficacia; pertinencia; entorno institucional; aspectos sociales y culturales; y, aspectos ambientales. Se recabó la opinión de una muestra de campesinos comuneros de la zona de estudio, considerando tanto a aquellos sectores que ya están siendo beneficiados de las obras de riego, cuanto a aquellos que continúan a la expectativa de ser tomados en cuenta. Se adoptó una escala de ponderación para asignar una categoría específica a cada indicador: Deficiente (1 a 3); Insuficiente (3,1 a 5); Regular (5,1 a 7); Bueno (7,1 a 9); Excelente (9,1 a 10).

\subsection{Eficacia}

Los resultados obtenidos luego del procesamiento de la información, proveniente tanto de los talleres participativos como de la aplicación del respectivo formato a los informantes calificados, se presentan en la Tabla 1. Como lo señala Menacho (2007), eficacia es un concepto que revela en qué medida un programa o una organización cumple sus objetivos. También puede referirse al logro estricto de un objetivo formulado en un período de tiempo determinado, sin tomar en cuenta el importe económico. La eficacia tiene que ver con las posibilidades de lograr el cumplimiento de los objetivos trazados por una organización sin considerar los costos. El criterio para abordar los aspectos de eficacia se basó en tres indicadores: beneficios directos para la población; incremento en la superficie regada; y, disminución en la explotación de acuíferos. La valoración de la eficacia del trasvase Chongón San Vicente, se sitúa en un promedio de $3,47 / 10$, equivalente a insuficiente. Esto significa que las familias reconocen como insuficiente el nivel de beneficios directos que han obtenido con la implementación del trasvase Chongón San Vicente.

Tabla 1. Valoración de los indicadores de eficacia del trasvase Chongón San Vicente

\begin{tabular}{cccc}
\hline Criterio & Indicadores & Valor & Promedio \\
\hline & $\begin{array}{c}\text { Incremento } \\
\text { ingresos }\end{array}$ & 3,19 & \\
Pertinencia & $\begin{array}{l}\text { Incremento } \\
\text { producción } \\
\text { alimentos } \\
\text { consumo local }\end{array}$ & 4,31 & 3,83 \\
& $\begin{array}{l}\text { Incremento } \\
\text { capacitación } \\
\text { adultos }\end{array}$ & 3,99 \\
\hline
\end{tabular}


La población local seguramente esperaba que el acceso al agua para consumo humano y riego, el incremento de la producción agropecuaria, la generación de empleo, el incremento de ingresos, entre otros, eran los beneficios directos. Sin embargo, hasta la fecha, los impactos positivos del trasvase continúan siendo una promesa.

\subsection{Pertinencia}

Los indicadores que evalúan la pertinencia del trasvase Chongón San Vicente fueron planteados de la siguiente manera: incremento de ingresos; incremento de producción de alimentos de consumo local; e incremento de capacitación de adultos (Tabla 2). Según Gurtler et al. (2010, p. 33), la pertinencia o relevancia busca valorar si una determinada iniciativa contribuye significativamente a los procesos de progreso y desarrollo, ya sea en el ámbito interno como en el contexto general en que se desenvuelve. En contraste, González (2005, p. 52) destaca que la pertinencia se puede valorar averiguando si el proyecto una vez finalizado respondió a las necesidades y al contexto de los grupos de hombres y mujeres participantes.

Tabla 2. Valoración de los indicadores de pertinencia del trasvase Chongón San Vicente

\begin{tabular}{llll}
\hline Criterio & Indicadores & Valor & Promedio \\
\hline & $\begin{array}{c}\text { Beneficios } \\
\text { del trasvase }\end{array}$ & 3,68 & \\
& $\begin{array}{l}\text { Incremento } \\
\text { áreas bajo }\end{array}$ & 3,54 & 3,47 \\
Eficacia & riego & & \\
& $\begin{array}{c}\text { Disminución } \\
\text { explotación } \\
\text { acuíferos }\end{array}$ & 3,19 & \\
& &
\end{tabular}

La pertinencia del trasvase Chongón San Vicente, considerando sus tres indicadores, fue valorado por los participantes con un valor promedio de 3,83/10 equivalente a insuficiente, tal como lo indica la Tabla 2. Esta valoración muestra hasta qué nivel ha contribuido el trasvase a mejorar el nivel de vida de las comunidades locales y hasta qué punto impactó en el contexto local.

\subsection{Entorno institucional}

Los indicadores del entorno institucional que estructuran este campo son: participación de organizaciones comunitarias; información de costos del trasvase; y, participación de gobiernos autónomos descentralizados. Hombres y mujeres pertenecientes a las comunas del valle del río Javita, valoraron en 4,60/10 el entorno institucional del trasvase Chongón Santa Elena, calificación que equivale a regular (Tabla 3). El entorno institucional, en general, puede verse afectado por diversos factores entre los que se puede mencionar los elementos ambientales, socioculturales y de género, los aspectos institucionales y las políticas de apoyo (Viñas, 2005, p. 103). Por otro lado, el régimen de competencias definido por la Constitución, el COOTAD (Asamblea Nacional, 2010, p. 22) y las resoluciones del Consejo Nacional de Competencias determinan que los gobiernos provinciales tienen la competencia exclusiva de planificar, construir, operar y mantener sistemas de riego en el ámbito de su jurisdicción.

Tabla 3. Valoración de los indicadores de entorno institucional

\begin{tabular}{|c|c|c|c|}
\hline Criterio & Indicadores & Valor & Promedio \\
\hline \multirow{3}{*}{$\begin{array}{c}\text { Entorno } \\
\text { institucional }\end{array}$} & $\begin{array}{c}\text { Participación } \\
\text { organizaciones } \\
\text { comunitarias }\end{array}$ & 6,68 & \multirow{3}{*}{4,60} \\
\hline & $\begin{array}{l}\text { Información } \\
\text { costos del } \\
\text { trasvase }\end{array}$ & 2,42 & \\
\hline & $\begin{array}{l}\text { Participación } \\
\text { GADs }\end{array}$ & 4,69 & \\
\hline
\end{tabular}

Lo que se revela con los resultados obtenidos es que tanto las comunas, cuanto las juntas de regantes en proceso de formación, fueron tomadas parcialmente en cuenta para el proceso de discusión del diseño de las obras del trasvase $(6,68)$; sin embargo hubo debilidad en el proceso de socialización de información relacionada con los costos de las obras hacia la población involucrada $(2,42)$; la participación de los GAD no ha tenido los niveles de preponderancia que se esperaba; muchas de las inversiones y decisiones en materia de riego y drenaje continuaron en manos del Estado central $(4,69)$. 


\subsection{Aspectos sociales y culturales}

Los indicadores relacionados con los aspectos sociales y culturales son: respeto de la cultura comunera; respeto de las tierras comunales; y, fortalecimiento de las organizaciones comunitarias (Tabla 4). Este criterio ha merecido la más alta valoración en promedio, en comparación con las otras mediciones, llegando a un 5,91/10, que equivale, de acuerdo a la tabla de ponderación a regular.

Tabla 4. Valoración de los indicadores sociales y culturales

\begin{tabular}{cccc}
\hline Criterio & Indicadores & Valor & Promedio \\
\hline & $\begin{array}{c}\text { Respeto cultura } \\
\text { comunera }\end{array}$ & 5,81 & \\
$\begin{array}{c}\text { Indicadores } \\
\text { sociales y } \\
\text { culturales }\end{array}$ & $\begin{array}{c}\text { Respeto tierras } \\
\text { comunales }\end{array}$ & 6,75 & 5,91 \\
& $\begin{array}{c}\text { Fortalecimiento } \\
\text { organizaciones } \\
\text { comunitarias }\end{array}$ & 5,18 & \\
\hline
\end{tabular}

Lo que puede afirmarse es que, hasta el momento, las prácticas de la cultura comunera han sido tomadas en cuenta parcialmente en el proceso de implementación del trasvase $(5,81)$. Al respecto, conviene señalar algunas prácticas vigentes de la cultura comunera, que aún se puede evidenciar en territorios rurales de Santa Elena: toma de decisiones en asamblea, rendición pública de cuentas, solidaridad y reciprocidad y no solo finalidad de lucro, negociación concertada en vez del conflicto, entre otras. Por otro lado, hasta el momento de la realización del estudio, los participantes consideran que, en buena medida, la propiedad de las tierras comunales ha sido respetada $(6,75)$. En promedio, los indicadores sociales y culturales han merecido una valoración de 5,18.

\subsection{Aspectos ambientales}

El manejo adecuado del ambiente alude a la habilidad de las sociedades humanas de lograr prosperidad, progreso y crecimiento económico perdurables en el tiempo, sin descuidar al mismo tiempo el ciclo de renovación de los recursos naturales y la calidad de vida de las actuales y futuras generaciones (Sepúlveda, 2016). En cuanto a los aspectos ambientales, se han analizado tres indicadores: conservación de suelos y fuentes de agua; disminución en los niveles de contaminación con agroquímicos; $\mathrm{y}$, disminución de impactos ambientales causados por el proceso de construcción e implementación del trasvase (Tabla 5). La valoración de este criterio se situó en un 2,49/10, equivalente a insuficiente.

Tabla 5. Valoración de los indicadores ambientales

\begin{tabular}{cccc}
\hline Criterio & Indicadores & Valor & Promedio \\
\hline & $\begin{array}{c}\text { Conservación } \\
\text { suelos y fuentes } \\
\text { de agua }\end{array}$ & 4,75 & \\
Aspectos & $\begin{array}{c}\text { Disminución } \\
\text { contaminación } \\
\text { químicos }\end{array}$ & 0,14 & 2,49 \\
& $\begin{array}{c}\text { Disminución } \\
\text { impactos } \\
\text { ambientales }\end{array}$ & 2,58 & \\
& \\
\hline
\end{tabular}

Uno de los efectos evidentes de la puesta en riego de nuevas superficies de tierra, tiene que ver con el desbroce y destrucción de la cubierta vegetal original y sus consecuencias en los ecosistemas locales. De acuerdo a la apreciación de la población local, los efectos del trasvase sobre los ecosistemas locales son de un nivel intermedio $(4,75)$; de otro lado, la población ha evidenciado que el uso de agroquímicos se ha ido incrementando sustantivamente en los territorios servidos con riego, particularmente el cultivo de maíz, que más se ha extendido en el territorio, implica la aplicación de un paquete tecnológico basado en insumos externos $(0,14)$. Finalmente, cuando se construye una obra de infraestructura de esta magnitud, los impactos que ocasionan las excavaciones y movimientos de tierra, así como la tala de vegetación natural alteran el paisaje y los ecosistemas, lo cual no ha sido atenuado con planes de mitigación y por ello su valoración $(2,58)$.

\subsection{Valoración general}

En cuanto a los efectos del trasvase Chongón San Vicente, se puede concluir que los aspectos sociales y culturales han merecido la mayor puntuación (Figura 2). Ello revela que la vigencia de las 
organizaciones sociales y la institucionalidad de la provincia de Santa Elena mantienen un nivel de legitimidad considerable, en relación con los otros indicadores. En contraste, los aspectos ambientales son los que más bajo nivel de ponderación han merecido por parte de la población consultada. En particular, la valoración más baja entre todos los indicadores $(0,14)$ en relación con la pregunta de si ha disminuido la contaminación con agroquímicos en las actividades productivas agropecuarias generadas por el trasvase, revela la percepción de la población respecto del paquete tecnológico que se está implementando en los sistemas de producción bajo riego. En promedio, los efectos del trasvase Chongón San Vicente sobre el entorno económico, social cultural y ambiental del valle del río Javita se ubica en un valor de 4,06/10, revelando que ha sido reprobado según el criterio de la población consultada.

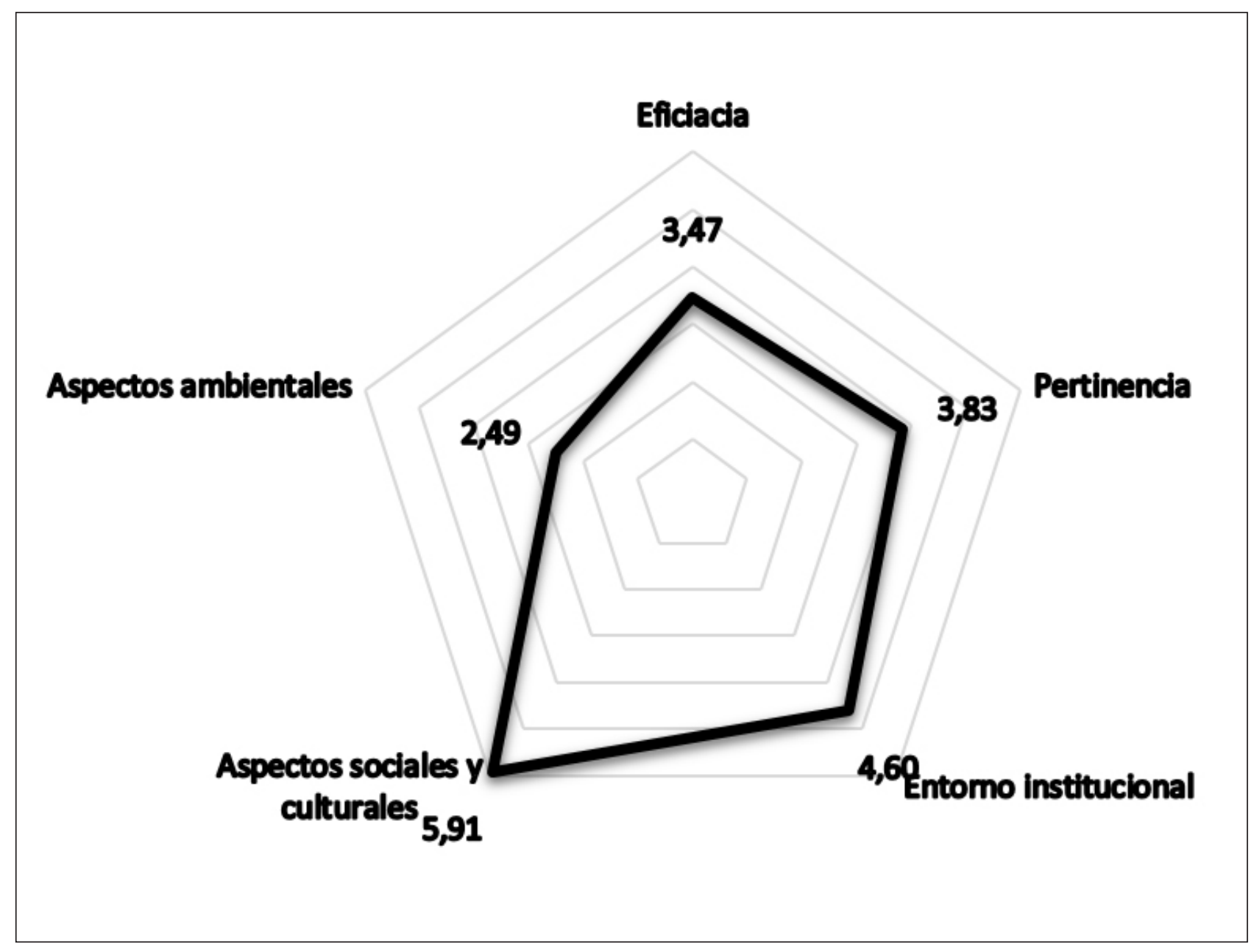

Figura 2. Efectos del trasvase Chongón San Vicente.

\subsection{Sistemas de producción}

\section{Tipo de productores identificados}

Conceptualmente, un sistema de producción podría ser definido como un complejo de actividades agropecuarias y extra agropecuarias ejecutadas por las familias campesinas, cuyo propósito es asegurar la reproducción de la unidad productiva, familiar, mediante el aprovechamiento racional de medios de producción, tales como el capital y la tierra, así como de la mano de obra existentes en un entorno ambiental, social y económico determinado (Apollin \& Eberhart, 1998, p. 66). De la sistematización del trabajo de campo se determinó la existencia de cinco tipos de sistemas de producción, que se detallan en la Tabla 6. 
Tabla 6. Sistemas de producción, principales características

\begin{tabular}{|c|c|c|}
\hline Tipo & $\begin{array}{l}\text { Tipo de } \\
\text { campesino }\end{array}$ & $\begin{array}{l}\text { Indicadores } \\
\text { económicos }\end{array}$ \\
\hline Tipo A & $\begin{array}{l}\text { Campesinos } \\
\text { proletarizados, } \\
\text { asalariados }\end{array}$ & $\begin{array}{c}\text { Ingreso familiar USD } \\
9.095 / \text { año; } \\
\text { Ingreso por persona } \\
\text { USD 13,78/día. }\end{array}$ \\
\hline Tipo B & $\begin{array}{c}\text { Campesinos que } \\
\text { implementan cultivos } \\
\text { y crianzas con acceso } \\
\text { al riego }\end{array}$ & $\begin{array}{l}\text { Ingreso familiar USD } \\
\text { 7.766/año; } \\
\text { Ingreso por persona } \\
\text { USD 10,38/día. }\end{array}$ \\
\hline Tipo $C^{*}$ & $\begin{array}{c}\text { Campesinos que } \\
\text { implementan cultivos } \\
\text { y crianzas mayores sin } \\
\text { acceso al riego }\end{array}$ & $\begin{array}{l}\text { Ingreso familiar USD } \\
6.728 / \text { año; } \\
\text { Ingreso por persona } \\
\text { USD 8,99/día. }\end{array}$ \\
\hline Tipo D & $\begin{array}{c}\text { Campesinos } \\
\text { exclusivamente } \\
\text { agrícolas con riego }\end{array}$ & $\begin{array}{l}\text { Ingreso familiar USD } \\
7.712 / \text { año; } \\
\text { Ingreso por persona } \\
\text { USD 10,62/día. }\end{array}$ \\
\hline Tipo E** & $\begin{array}{c}\text { Campesinos que } \\
\text { implementan o cultivos } \\
\text { o crianzas mayores sin } \\
\text { acceso al riego }\end{array}$ & $\begin{array}{l}\text { Ingreso familiar USD } \\
7.541 / \text { año; } \\
\text { Ingreso por persona } \\
\text { USD 10,39/día. }\end{array}$ \\
\hline
\end{tabular}

*Los campesinos Tipo C, no disponen de riego, sin embargo, trabajan en agricultura y ganadería.

** Los campesinos Tipo E, sí disponen de riego y trabajan solamente en agricultura o solamente en ganadería.

A continuación, se describen los cinco tipos de sistemas de producción identificados:

- Tipo A. Los productores del Tipo A disponen de tierra en posesión, pero no la trabajan debido a que encuentran mejores oportunidades en labores distintas a la agropecuaria. La totalidad de los ingresos de los campesinos de este grupo provienen de actividades extra-agropecuarias. Trabajan fuera de su parroquia e incluso en provincias vecinas en labores de pesca, construcción y servicios. $\mathrm{Su}$ ingreso promedio es el más elevado entre todos los demás casos estudiados.
- Tipo B. Aprovechando el acceso al agua de riego, los campesinos identificados con el sistema Tipo B se dedican a cultivos y crianzas. Al interior de la unidad productiva manejan pequeños lotes con diversidad de cultivos y animales menores. Ganado vacuno y caprino se maneja en pastoreo a campo abierto. Tan solo el $20 \%$ de los ingresos proviene de la actividad agropecuaria. El ingreso más elevado ( $80 \%$ ) proviene de actividades extra-agropecuarias efectuadas fuera de la finca, o incluso fuera de su territorio.

- Tipo C. Los campesinos del sistema Tipo C no disponen de acceso al riego; trabajan con cultivos y crianzas en función de las precipitaciones pluviales. A pesar de la restricción hídrica, mantienen una considerable cantidad de plantas y animales dentro de la finca y en el traspatio de la casa. Solamente un $25 \%$ de la tierra está siendo trabajada. De manera similar al caso anterior, solo un $17 \%$ del ingreso familiar proviene de actividades agropecuarias. La mayor proporción de sus ingresos proviene de actividades extra-agropecuarias, fuera de la finca e incluso fuera de la parroquia.

- Tipo D. Los campesinos del Tipo D emplean el agua de riego para realizar cultivos, utilizando la mayoría del terreno; la particularidad de este sistema es que no invierten recursos en crianzas. De los sistemas que trabajan en actividades agropecuarias, es el que muestra el más alto porcentaje de ingresos obtenidos de la actividad agropecuaria con casi un $45 \%$ del total de ingresos. Su dedicación más importante es la agricultura de ciclo corto, aunque siguen obteniendo el mayor porcentaje de sus ingresos de actividades extra agropecuarias. Un $90 \%$ del terreno de estos productores está en la figura de posesión.

- Tipo E. Los campesinos del Tipo E, que no disponen de riego, realizan sus labores agropecuarias de acuerdo con la precipitación pluvial. Se dedican alternativamente a cultivos o a crianzas. Una pequeña parte del terreno es trabajado. Sus indicadores de productividad son los más bajos de todos los sistemas analizados. Apenas un $8,7 \%$ proviene de actividades agropecuarias. 


\subsection{Ingresos familiares}

Al abordar el análisis de los ingresos familiares, se revelan aspectos clave de los sistemas de producción estudiados. El rubro otros ingresos representa el mayor aporte de los ingresos totales. Se puede observar que las actividades extra-agropecuarias generan la mayor cantidad de ingresos para la totalidad de casos estudiados, muy por encima de los ingresos agropecuarios netos, como lo muestra la Tabla 6 . En las condiciones de la zona de estudio, las personas ejercen la pluriactividad, que se ha convertido en el mecanismo a través del cual los habitantes rurales buscan superar sus dificultades económicas, obtener ingresos, pero -y esto es lo importante- sin abandonar su relación con su tierra, con su cultura, con su unidad de producción familiar (Bermeo, 2017, p. 28). A más del trabajo en la finca, las actividades extra-agropecuarias sirven como mecanismo para mejorar sus ingresos y elevar su nivel de vida.

Tabla 7. Ingreso total vs. Otros ingresos

\begin{tabular}{lrrrrr}
\hline \multicolumn{1}{c}{ Indicador } & \multicolumn{1}{c}{ Tipo A } & \multicolumn{1}{c}{ Tipo B } & Tipo C & Tipo D & \multicolumn{1}{c}{ Tipo E } \\
\hline IAN * & 0 & 1.601 & 1.147 & 3.449 & 656 \\
Otros ingresos & 9.095 & 6.165 & 5.581 & 4.263 & 6.884 \\
Ingreso total & 9.095 & 7.766 & 6.728 & 7.712 & 7.540 \\
Porcentaje OI/IT** & $100 \%$ & $79 \%$ & $83 \%$ & $55 \%$ & $91 \%$ \\
Porcentaje IAN/IT** & $0 \%$ & $21 \%$ & $17 \%$ & $45 \%$ & $9 \%$ \\
\hline *IAN: Ingreso Agropecuario Neto & & & & \\
**OI/OT Otros Ingresos / Ingreso Total & & & & \\
***IAN/IT: Ingreso Agropecuario Neto / Ingreso & & & & \\
Total &
\end{tabular}

Conviene señalar que, como lo indica Bermeo (2017, p. 28), en las condiciones de la zona de estudio, las personas ejercen la pluriactividad, que se ha convertido en el mecanismo a través del cual los habitantes rurales buscan superar sus dificultades económicas, obtener ingresos, pero $-\mathrm{y}$ esto es lo importante- sin abandonar su relación con su tierra, con su cultura, con su unidad de producción familiar. Varios elementos han alentado la existencia de la pluriactividad en la zona estudiada: el exiguo ingreso agrícola; el deficiente acceso a la tierra y al servicio de riego; la inestabilidad de la agricultura que depende de la estacionalidad de las precipitaciones; la existencia de oportunidades de empleo en otros sectores y regiones, entre otros factores (Bermeo, 2017, p. 51).

\subsection{Ingresos totales en relación con la canasta básica}

Comparando las cifras de los diferentes sistemas de producción analizados, se concluye que el ingreso total en el sistema Tipo A, supera tanto el ingreso familiar mensual tipo, como también el costo de la canasta familiar básica (Tabla 7). En los demás sistemas de producción, los ingresos totales, que son la suma del ingreso agropecuario neto más el rubro otros ingresos, no logran cubrir los valores de la canasta básica ni alcanzan el valor del ingreso familiar mensual tipo ${ }^{1}$.

Los productores agrupados en Tipo B y Tipo D son los que mejores ingresos totales muestran, cubriendo entre el $90 \%$ y $91 \%$ del valor de la canasta básica; debe tomarse en cuenta que el ingreso total es la suma del ingreso extra-agropecuario más el ingreso agropecuario neto. Solamente los productores Tipo A superan el valor de la canasta básica con los ingresos obtenidos exclusivamente de fuentes externas a la unidad productiva. Es decir, ninguno de los sistemas de producción agropecuarios logra cubrir el valor de la canasta básica con el ingreso neto agropecuario.

1 Según el INEN, en el año 2018, el costo de la canasta familiar básica se ubicó en \$ 712,66, mientras que el ingreso familiar mensual de un hogar tipo fue de \$720,53. El Telégrafo (2018). 
Tabla 8. Ingresos totales y canasta básica

\begin{tabular}{lccccc}
\hline \multicolumn{1}{c}{ Indicador } & Tipo A & Tipo B & Tipo C & Tipo D & Tipo E \\
Ingreso total & 9.095 & 7.766 & 6.728 & 7.712 & 7.540 \\
Ingresos mensual & 758 & 647 & 561 & 643 & 628 \\
\% de la canasta básica & $106 \%$ & $91 \%$ & $79 \%$ & $90 \%$ & $88 \%$ \\
\hline
\end{tabular}

\subsection{Valorización del trabajo familiar}

Comparando las cifras, se concluye que el ingreso agropecuario anual (IAN) contribuye con no más del $29 \%$ al ingreso total (IT) en la mayoría de casos estudiados. En el caso del sis- tema D, el IAN representa un 55\% del IT. En el caso del sistema A, el ingreso agropecuario neto es cero (Tabla 8). Analizando las cifras por día, se puede apreciar que ninguno de los sistemas llega al monto de USD 15 que se paga por jornal diario trabajado.

Tabla 9. Ingresos familiares por año y día

\begin{tabular}{lccccc}
\hline Indicador & Tipo A & Tipo B & Tipo C & Tipo D & Tipo E \\
\hline IAN/UTF_DUF/año & 0 & 667 & 478 & 1.277 & 328 \\
IT/UTF/año & 3.032 & 2.284 & 1.979 & 2.337 & 2.285 \\
IAN/UTD_DUF/día & 0 & 3 & 2,2 & 5,8 & 1,5 \\
IT/UTF/día & 13,78 & 10,38 & 8,99 & 10,62 & 10,39 \\
Porcentaje IAN/IT & $0 \%$ & $29 \%$ & $24 \%$ & $55 \%$ & $14 \%$ \\
\hline
\end{tabular}

Los sistemas de producción que incluyen actividades agropecuarias como medio para generar ingresos no presentan perspectivas halagadoras para el futuro inmediato, a menos que se implementen acciones complementarias al riego con el fin de dinamizar la agricultura en este territorio. Incluso los sistemas Tipo B y Tipo D, que tienen acceso al riego, que invierten considerables recursos humanos, financieros y materiales y que están emprendiendo en actividades productivas agropecuarias de cierta intensidad, con empleo de mano de obra, insumos y que están relacionados con el mercado, no se constituyen en referentes de sistemas de producción rentables.

Por otro lado, es preciso comprender cómo se combinan los objetivos de la producción con los derechos de acceso al agua en las comunidades. No se trata solo de medir indicadores de eficiencia, sino incorporar también la visión de los productores en cuanto se refiere a la lógica agrotécnica de las familias. La dinámica de los sistemas de producción y sus capacidades de adaptación a las condiciones de acceso al riego son elementos determinantes en el análisis (Apollin \& Eberhart, 1998, p. 16).

\subsection{Elementos para un plan de gestión del riego a fa- vor de la agricultura campesina}

En la línea de proponer un modelo de gestión del riego, siguiendo a Anten \& Williet (2000, p. 6), se propone una subdivisión en tres subsistemas: a) Subsistema socio-organizativo, que comprende a los diversos usuarios, sus formas organizativas, así como los acuerdos y las normas que han adoptado para la gestión social del sistema de riego, incluyendo las actividades de operación y mantenimiento, así como la red de relaciones internas y externas; b) Subsistema infraestructural que viene a estar conformado por todo el complejo de obras de infraestructura de captación, conducción, almacenamiento, reparto y distribución del agua de riego en un territorio; y, c) Subsistema productivo, que no es otra cosa que el conjunto de ac- 
tividades productivas que se encuentra bajo riego; es decir, los cultivos, los animales, el manejo del agua y las técnicas del riego; la gestión del suelos en cada una y en la totalidad de las parcelas; las técnicas de manejo agronómico propiamente dicho, así como las actividades de postcosecha, transformación y comercialización; en suma, el manejo de la economía de la producción campesina que dispone de riego.

El Plan de Gestión constituye el diseño para lograr el mejor manejo de una organización en el proceso de la ejecución de sus actividades diarias y también en el mediano y largo plazo; comprende una serie de métodos cotidianos para el manejo de personas, recursos económicos, procesos organizativos, en conjunto con el quehacer filosófico de una organización (Centro para la Salud y Desarrollo de la Comunidad, 2019).

Del procesamiento de la información, se han obtenido los elementos que se describen a continuación, los cuales constituyen un marco orientador para lograr el propósito enunciado en este componente.

\subsection{Subsistema socio - organizativo}

\section{- Reparto del agua de riego}

Se consultó a los participantes respecto de cómo hacer el reparto del agua de riego que está llegando a los territorios comunales. Los criterios a escoger fueron: a) considerando la superficie actual de las posesiones dentro de cada territorio comunal; b) considerando superficies iguales para cada familia beneficiada (2 ha); c) tomando en cuenta la necesidad hídrica de los cultivos. Según la opinión de los productores consultados la necesidad hidrica de los cultivos es la que mayor valoración obtuvo. Es decir, el caudal que recibirá cada productor, según la opinión mayoritaria de los consultados, estará en función de la demanda del cultivo en la superficie que cada productor tenga en posesión, lo que puede generar una cierta tendencia a la diferenciación campesina al interior de las comunas.

El diseño de instituciones para la gestión del riego entraña múltiples pruebas y ajustes, una y otra vez, con la finalidad de aprender incesantemente respecto de cómo las reglas afectan la conducta de los participantes. La selección de qué tipo de instituciones no puede corresponder a una decisión única y definitiva, sino más bien debe convertirse en algo progresivo en un entorno de elevada incertidumbre (Ostrom, 1992, p. 45).

\section{- Administración, operación y mantenimiento.}

Las tareas de administración, operación y mantenimiento deben estar a caurgo de las juntas de regantes. Según este criterio, se desplaza a la comuna, como entidad matriz de organización social en el territorio, de estas funciones técnicas de la gestión del riego.

En un sistema de riego se administran los recursos físicos, económicos y humanos; la operación implica el manejo de todo el sistema, desde la captación hasta la entrega del agua al usuario; el mantenimiento supone que todos los elementos de la infraestructura se encuentren en condiciones adecuadas para un oportuno y adecuado servicio de riego (Concha, 2013, p. 10).

Es fundamental para que el sistema funcione y se mantenga en el tiempo que los usuarios participen en la formulación de al menos las más importantes decisiones operativas. Por otro lado, las normas que se adoptan y están vigentes en un contexto de tradición cultural vigente, constituyen normas de conocimiento compartido. Los sistemas que funcionan observando normas o creencias compartidas provenientes de raza, casta, religión, pertenencia étnica deben tomar muy en cuenta estos elementos (Ostrom, 1992, p. 53-57).

\section{- Organización del riego}

La organización del riego debe ser efectuada considerando la existencia de grupos de familias al interior de cada territorio comunal. Esta opción ha sido escogida como prioritaria frente a las otras planteadas: organizar el riego considerando a cada familia; o, hacerlo tomando en cuenta la situación del territorio comunal en su conjunto.

En ese sentido, hablar de sistemas de riego, como lo indica CAMAREN (2017, p. 27) implica concebir este servicio como un mecanismo para optimizar la producción agropecuaria y también la organización social; la puesta en marcha de nuevos sistemas debe abordarse como una construcción 
eminentemente social en la cual el Estado y las organizaciones participan en el diseño e implementación.

Ya se trate la organización a nivel de grupos de familias o comunidades, las reglas o normas contienen básicamente disposiciones para prohibir o permitir el curso de alguna acción o proceso; este conjunto de normas solo tiene sentido si los usuarios conocen de su existencia y tienen la expectativa de que todos funcionen de acuerdo a lo establecido en esa normativa (Ostrom, 1992, p. 22).

\subsection{Subsistema infraestructural}

\section{- Método de aplicación}

El método preferido de aplicación de agua en los cultivos debe ser el goteo. Esta opción apunta a la eficiencia en la aplicación del agua de riego, aunque queda la incertidumbre de cómo financiar los costos de inversión y mantenimiento en las condiciones de las fincas campesinas. La opción preferente por el goteo parece haber sido el resultado de una tendencia general por instalar este tipo de emisores en proyectos públicos y privados, sin el suficiente análisis. Se debe considerar que el riego por goteo puede ser una buena opción, dependiendo de las condiciones económicas del productor, del tipo de suelo, de los cultivos a instalarse y, sobre todo, del análisis beneficio - costo. Sin embargo, es preciso mencionar que debido a los escasos recursos de los que disponen las familias, de no darse la inversión del Estado y no encontrarse alternativas para disminuir los costos que demanda este tipo de sistema de aplicación, se corre el riesgo de que el riego por goteo no sea implementado, con la consecuente disminución de los resultados en los rendimientos y en la rentabilidad de la agricultura.

La elección del método de aplicación del riego tiene que ver, como lo señala CAMAREN (2017, p. 24), con el proceso de capacitación y asesoría que se demanda, tiene que contar con un elevado número de profesionales de diversos campos, tanto del área social y cultural, como del área técnica que favorezcan la comprensión de la gestión del riego como un mecanismo para la promoción social de los habitantes del campo, como una perspectiva para el desarrollo del territorio, como el espacio para recrear un modelo solidario, equitativo y sostenible de agricultura.

\section{- Instalación del sistema de riego}

La instauración de un sistema de riego, con todos sus componentes físicos, instalaciones y equipos debe hacerse considerando el ahorro de agua, por encima de su duración y costos de implementación y operación. Este criterio expresa la necesidad de evitar al máximo posible el pago por la tarifa del agua de riego. Aunque la instalación de un sistema de riego depende de las condiciones hidrológicas y de la situación socioeconómica de los regantes, el criterio de las personas consultadas es que se priorice el criterio de ahorro del agua al momento de diseñar e instalar un sistema de riego.

El tratamiento de la infraestructura de riego, como parte de un sistema, implica analizar la calidad de la obra, las condiciones que deben implementarse para una tarea de mantenimiento preventivo, así como de mantenimiento de emergencia. De lo que se trata es de evaluar la funcionalidad y la operación del sistema, además de analizar el área de riego en general, así como la superficie del área regable (Salazar et al., 2010, p. 16).

\subsection{Subsistema productivo}

\section{- $\quad$ Tipo de agricultura}

En cuanto al tipo de agricultura que debe ponerse en marcha una vez que se cuente con riego, se ha expresado una tendencia levemente mayoritaria por los cultivos de ciclo corto y los de carácter transitorio. Tanto los cultivos perennes, como la combinación de cultivos y animales, quedaron desplazados a lugares secundarios. Sin embargo, las preferencias no son marcadas. La valoración final deja entrever una cierta indecisión.

Indudablemente, un proceso de mejora en los modelos de riego campesino implica desarrollar prácticas de riego acordes con las especificidades de cada localidad, tomando muy en cuenta los aspectos socio-económicos y la cultura local de las familias usuarias; asimismo, conviene observar las condiciones de los sistemas de producción agrícola y pecuario, la rentabilidad que tienen, el tipo y las características de los suelos y los factores climáticos, entre otros factores (CAMAREN, 2017, p. 21). 


\section{- Destino de la producción bajo riego}

En consonancia con los demás aspectos, el destino de la producción bajo riego debe ser el mercado regional y nacional. Los productores están interesados en emprender en actividades rentables, hacer presencia en el mercado, obtener ganancias y generar empleo para sus familias.

Es necesario tener presente que la calidad de los alimentos que se consume está fuertemente atada al papel que juegan los agricultores en el manejo de los agroecosistemas, en la protección de las fuentes hídricas, en el cuidado que ponen para ahorrar equipos e insumos externos, en la utilización racional de las fuentes de energía; para cualquier mercado, en la actualidad, la calidad también tiene que ver con la manera como son producidos los alimentos, en manos de quienes está el proceso productivo y en qué lugares y condiciones se producen (CAMAREN, 2017, p. 16).

A continuación, en la Figura 3 se muestra un esquema de un Plan de Gestión que debe ser considerado al momento de discutir propuestas para el impulso de la agricultura bajo riego en este territorio. En cuanto al goteo como método de aplicación del riego, conviene destacar que este criterio seleccionado genera discusión debido a los escasos ingresos de los que disponen las familias campesinas. La Figura muestra en detalle la estructura de un plan de gestión del riego, como marco orientador de una política pública. Cada uno de los subsistemas contiene componentes y criterios orientadores, cada cual con sus respectivas aclaraciones. Se trata de una propuesta de política pública surgida de la opinión de los productores y organizada a partir del enfoque sistémico y constituye un aporte de la investigación para las organizaciones campesinas y las entidades oficiales.

Figura 3. Estructura del Plan de Gestión del Riego.

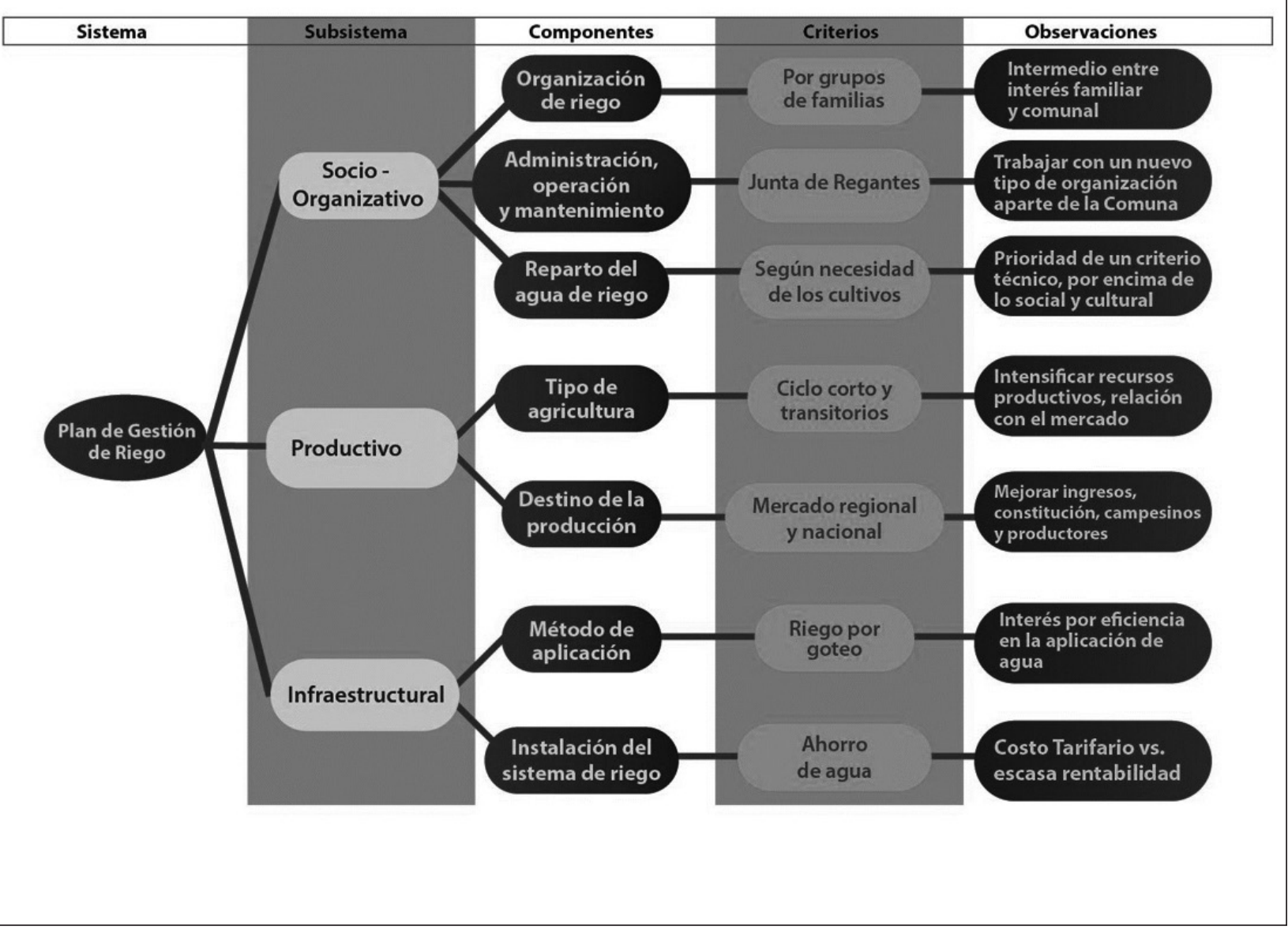




\section{Conclusiones}

En relación con los efectos del trasvase Chongón San Vicente

El proyecto Chongón San Vicente se planteó con la finalidad de trasvasar las aguas desde el canal Chongón Sube y Baja hasta la presa San Vicente, con la pretensión de incorporar a diversas comunidades al desarrollo integral del país; sin embargo, considerando la percepción de los habitantes de las comunas del valle del río Javita y tomando en cuenta la escala de ponderación mencionada en la Sección 3.1 de este artículo, el trasvase Chongón San Vicente: a) ha sido insuficiente en cuanto a aspectos relacionados con su eficacia, lo que revela el bajo nivel de cumplimiento de los objetivos trazados; b) ha resultado insuficiente en cuanto a aspectos de su pertinencia, si se toma en consideración la débil contribución de esta obra con los procesos de desarrollo de los pueblos asentados en su área de influencia; c) ha demostrado ser regular en cuanto se refiere al entorno institucional, en la medida en la cual los procesos organizativos y de gestión se han mantenido en un nivel regular, al igual que las capacidades de los sectores involucrados; d) ha obtenido una ponderación de regular en lo referente a aspectos de orden social y cultural, si se observa que la equidad y la disminución de la pobreza para los sectores de población influenciados por esta obra se han mantenido sin mayor variación; $\mathrm{y}, \mathrm{e}$ ) se ha mostrado deficiente en relación con la sustentabilidad ambiental, en cuanto se refiere al manejo que se ha hecho de los recursos naturales y al aumento de la contaminación con agrotóxicos.

\section{En relación con los sistemas de producción}

Distribuidos en las dos zonas homogéneas, se ha podido establecer la existencia de 5 tipos de sistemas de producción: a) Sistema de producción Tipo A, extra agropecuario sin riego, al cual corresponde un tipo de campesino asalariado, siendo el único que no realiza actividades agropecuarias; b) Sistema de producción Tipo B, al cual se corresponde un tipo de campesino productor agrícola y pecuario con acceso al riego proveniente del trasvase o de acuífero de recarga; c) Sistema de producción Tipo $\mathrm{C}$, al que se corresponde un tipo de campesino que implementa cultivos y crianzas sin contar con acceso al agua de riego; d) Sistema de producción Tipo $\mathrm{D}$, correspondiente con un tipo de campesino productor agrícola con acceso al agua de riego; y, d) Sistema de producción Tipo E que trabaja una de las dos actividades: agricultura o crianza de animales, pero sin acceso al riego.

Al abordar el análisis de los ingresos familiares, el rubro Otros Ingresos, proveniente de actividades extra-agropecuarias es mucho más alto que el rubro de Ingresos Agropecuarios Netos en todos los casos. Las actividades extra-agropecuarias generan la mayor cantidad de ingresos para la totalidad de casos estudiados. Los ingresos provenientes de actividades extra-agropecuarias del sistema Tipo A superan al ingreso familiar mensual de un hogar tipo, así como también el costo de la canasta familiar básica. En los demás sistemas de producción, los Ingresos Totales no logran cubrir los valores de la canasta básica. Los ingresos agropecuarios contribuyen de manera marginal al Ingreso Total en la mayoría de casos estudiados; solamente para el sistema Tipo $\mathrm{D}$, el ingreso agropecuario es más de la mitad del Ingreso Total.

Todos los sistemas de producción agropecuarios presentan limitaciones en su viabilidad económica y productiva. Incluso los sistemas Tipo B y D, que tienen acceso al riego, no constituyen referentes de viabilidad.

La baja rentabilidad de las unidades de producción analizadas, el deficiente nivel de ingresos que obtienen los campesinos que trabajan en sus fincas, y fundamentalmente la carencia de otros servicios de apoyo a la producción, no dejan ver una perspectiva prometedora, incluso con la puesta en marcha definitiva del trasvase. La construcción de las obras rezagadas del trasvase, por sí sola, no elevará la productividad ni la calidad de vida de las familias campesinas y las comunas que trabajan en el valle del río Javita.

\section{Lineamientos para un plan de gestión del riego}

Para concebir un plan de gestión del riego en la zona de estudio, se propone una subdivisión en tres subsistemas: subsistema socio-organizativo; subsistema infraestructural; $\mathrm{y}$, subsistema productivo.

En cuanto al subsistema socio-organizativo, la organización del riego debe ser efectuada 
considerando a los grupos de familias existentes al interior de los territorios comunales. Las actividades de administración operación y mantenimiento deben ser llevadas a cabo por las juntas de regantes. El reparto de agua de riego debe hacerse fundamentalmente atendiendo a las necesidades hídricas de los cultivos.

En relación con el subsistema infraestructural, a pesar de que la tendencia preferencial de los agricultores es hacia el goteo, es necesario considerar la realidad de los ingresos de los agricultores, lo que no permitirá que por sí solos estén en capacidad de invertir en su implementación. La instalación de todos los componentes de un sistema de riego deberá atender como prioridad al propósito de ahorrar agua.

\section{Referencias}

Álvarez, S. (2002). Etnicidades de la Costa ecuatoriana. Quito, Ecuador: Abya-Yala.

Anten, M., \& Willet, H. (2000). Guía para el diagnóstico enfocado de sistemas de riego DER. Cajamarca: Proyecto de Cooperación PRONAMACHCS - SNV.

Apollin, F., \& Eberhart, Ch. (1998). Metodologías de análisis y diagnóstico de sistemas de riego campesino. Quito: CAMAREN. CESA. CICDA - RURALTER.

Asamblea Nacional. Código Orgánico de Organización Territorial, Autonomías y Descentralización. (2010). Quito, Ecuador.

Bermeo, M. (2017). La pluriactividad y su contribución a la calidad de vida de los territorios rurales: El caso de la parroquia de Colonche en la provincia de Santa Elena. Quito: Facultad Latinoamericana de Ciencias Sociales.

CAMAREN. (2017). El desarrollo de la agricultura bajo riego. Foro de los Recursos Hídricos. Mesas de Trabajo Nacional. Quito: CAMAREN.

Centro para la Salud y Desarrollo de la Comunidad (2019). Caja de Herramientas de Comunita-
Haciendo referencia al subsistema productivo, el tipo de agricultura que debe practicarse en el territorio una vez que se cuente con riego permanente del trasvase, muestra una tendencia predominante hacia la implementación de cultivos de ciclo corto y transitorio. Finalmente, el destino de la producción bajo riego debe ser orientado hacia el mercado nacional y regional. Esta es la opinión mayoritaria, aunque también se ha expresado un considerable porcentaje en el sentido de que la producción bajo riego debería ser pensada con fines de exportación.

Para que el riego se convierta en un elemento dinamizador de la economía local, no basta con obras de infraestructura. Es indispensable que el Estado invierta en un programa de desarrollo rural inclusivo, equitativo y eficiente

rias. Universidad de Kansas. Obtenido de: https://ctb.ku.edu/es

Concha, M. (2013). Manual de Administración, Operación y Mantenimiento del Sistema de Riego. Primera Edición. La Paz. Bolivia.

Empresa Pública del Agua. (2014). Proyecto Trasvase Chongón Sube y Baja a la Presa San Vicente en el cantón Santa Elena de la provincia de Santa Elena. Guayaquil: Empres Pública del Agua.

GADPSE, Gobierno Autónomo Descentralizado Provincial de Santa Elena. (2016). Plan Provincial de Riego y Drenaje para la Producción y Desarrollo de Santa Elena. Versión preliminar. Santa Elena: GADPSE.

González, L. (2005). La evaluación en la gestión de proyectos y programas de desarrollo: una propuesta integradora en agentes, modelos y herramientas. Vitoria-Gasteiz: Servicio Central de Publicaciones del Gobierno Vasco.

Gurtler, G., Bain, C., \& Shikiya, H. (2010). Glosario de términos para el fortalecimiento de capacidades en procesos estratégicos. Buenos Aires: Centro Regional Ecuménicos de Asesoría y Servicio. 
Menacho, L. (2007). Glosario básico de gestión de proyectos. Obtenido de: https://www.gestiopolis. com/glosario-basico-de-gestion-de- proyectos.

Ostrom, E, (1992). Diseño de instituciones para sistemas de riego auto-gestionarios. Traducción de Adriano Miguel Tejada. San Francisco: Centro Internacional para la Autogestión, Institute for Contemporary Studies.

Salazar, L., Saravia, R., \& Rafael, R., (2010). Sustentabilidad y autogestión de sistemas de riego. Cochabamba: PROAGRO. BMZ. GTZ
Sepúlveda, J. (2016). Sustentabilidad ambiental: referente esencial para el desarrollo regional. Revista Producción + Limpia, 11(2), 7-8.

Viñas, V. (2005). Conceptos clave de seguimiento y evaluación de programas y proyectos. Breve Guía. Lima: Fondo Internacional de Desarrollo Agrícola (FIDA). División para América Latina y El Caribe. 